\title{
Um modelo lógico da Rede Cegonha
}

\section{1 Pauline Cristine da Silva Cavalcanti, ${ }^{2}$ Garibaldi Dantas Gurgel Junior, ${ }^{3}$ Ana Lúcia Ribeiro de Vaconcelos, ${ }^{4}$ André Vinicius Pires Guerrero I}

Resumo: O objetivo deste artigo foi construir um Modelo Lógico (ML) da "Rede Cegonha" (RC), lançada pelo Ministério da Saúde em 2011, visando garantir o atendimento qualificado a gestantes e crianças com até dois anos de idade. A RC parte do diagnóstico de que as morbimortalidades materna e infantil permanecem elevadas, com prevalência da medicalização do nascimento e uso de tecnologias sem evidências científicas e que não consideram a gestante como protagonista do processo de gestação e parto. Foi realizada pesquisa em documentos oficiais do ministério e em outras fontes documentais, entre fevereiro e outubro de 2011. A elaboração do ML deu-se em três etapas: resposta a 12 "perguntaschave” para modelagem de programas; explicação do problema e referências básicas; e construção do ML da RC com base em duas referências em modelagem de programas: Bezerra, Cazarin e Alves (2010) e Cassiolato e Gueresi (2010). Observaram-se lacunas na lógica da RC, o que aponta para a necessidade de rever a teoria dessa estratégia com vistas a aumentar suas chances de sucesso. O ML mostra-se útil como uma ferramenta de gestão, com papel de explicitar a $\mathrm{RC}$ de forma prática e clara e auxiliar o processo de comunicação e divulgação, e pode ser utilizado na organização dos trabalhos de avaliação.

> Palavras-chave: mortalidade materna; mortalidade infantil; Modelo Lógico; Rede Cegonha.

\author{
1 Enfermeira; mestranda \\ em Saúde Coletiva pela \\ Universidade de Brasília. \\ Departamento de Atenção \\ Básica do Ministério da Saúde, \\ Brasília, Brasil. Endereço \\ eletrônico: pauline.cavalcanti@ \\ gmail.com \\ ${ }^{2}$ Médico; PhD em Políticas \\ Públicas pela Universidade \\ de Manchester; docente e \\ pesquisador do Centro de \\ Pesquisas Aggeu Magalhães \\ (CpqAM) em Recife, e da \\ Fiocruz,Brasília, Brasil. Endereço \\ eletrônico: gurgel@cpqam. \\ fiocruz.br \\ ${ }^{3}$ Doutora em Ciências da Saúde \\ pela Universidade de Brasília; \\ pesquisadora do CpqAM/ \\ Fiocruz/Ministério da Saúde. \\ Brasília, Brasil. Endereço \\ eletrônico: analucia@cpqam. \\ fiocruz.br \\ ${ }^{4}$ Mestre em Saúde Pública \\ pelo CpqAM/Fiocruz; analista \\ de Gestão em Saúde da \\ Fiocruz. Endereço eletrônico: \\ andreguerrero@fiocruz.br
}

Recebido em: 08/05/2013 Aprovado em: 03/12/2013 


\section{Introdução}

Em 1990, a Taxa de Mortalidade Materna (TMM) no Brasil atingia 140 óbitos por 100.000 nascidos vivos. Após mais de uma década e meia de esforços, em 2007, o indicador apresentou uma taxa de 75 óbitos por 100.000 nascidos vivos. Embora essa taxa tenha sofrido uma redução de $52 \%$ nesse período, o número de mortes maternas no Brasil permanece elevado (BRASIL, 2010). Nesse contexto, há mais de duas décadas discute-se o modelo de atenção ao parto no Brasil com o objetivo de atingir padrões aceitáveis, aproximando-se das taxas alcançadas em outros países. No Brasil, esse modelo vem sendo construído com base na experiência dos profissionais que trabalham nesse campo e nas iniciativas da humanização de atenção ao parto e ao nascimento em instituições de saúde que se tornaram referência no país (BRASIL, 2011g; RATTNER, 2009).

A partir de 2000, esse modelo começou a ser sistematizado em marcos normativos e manuais técnicos do Ministério da Saúde (MS) para o Sistema Único de Saúde (SUS) (BRASIL, 2011g). Apesar de se observarem avanços que se refletem nos resultados obtidos na saúde da mulher e da criança, esse processo tem-se dado de forma muito lenta (BRASIL, 2009).

Atualmente, organismos de saúde internacionais e nacionais preconizam um modelo de atenção ao parto e ao nascimento que proporcione às gestantes, às puérperas e aos recém-nascidos uma assistência humanizada e de qualidade (BRASIL, 2011g; 2001; DINIZ, 2005). Nesse modelo, são centrais a garantia do acesso às práticas de saúde baseadas em evidências científicas e o reconhecimento da gestante e de seus familiares como "atores principais" nessa cena, e não "espectadores" (DINIZ, 2005).

O MS alerta que, principalmente a partir de 2001, houve diminuição da velocidade de queda no indicador de mortalidade materna. Assim, são necessários maiores esforços para atingir valor igual ou inferior a 35 óbitos por 100.000 nascidos vivos até 2015, meta determinada no quinto Objetivo do Milênio (ODM) (BRASIL, 2010).

Em relação à mortalidade infantil, houve decréscimo de cerca de $60 \%$ desses óbitos nas últimas duas décadas. Em 1990, foram 57,1 óbitos por 1.000 nascidos vivos; em 2008, esse número diminuiu para 19 óbitos por 1.000 nascidos vivos. Os resultados apontam que o Brasil conseguirá cumprir o quarto ODM até 2015: 
redução em dois terços da Taxa de Mortalidade Infantil (TMI) (BRASIL, 2010).

Entretanto, a redução das mortes ocorreu principalmente no componente pósneonatal, fazendo com que a mortalidade neonatal seja o principal componente da mortalidade infantil.

Vale ressaltar que, mesmo com os avanços observados em todo o país, as regiões Nordeste e Norte ainda concentram TMI muito elevadas, contribuindo de maneira importante para a menor velocidade de redução do indicador (BRASIL, 2010).

Desde o surgimento do Programa de Humanização no Pré-Natal e Nascimento (BRASIL, 2000), da Política Nacional de Atenção Integral à Saúde da Mulher (BRASIL, 2004a) e do Pacto Nacional pela Redução da Mortalidade Materna e Neonatal (BRASIL, 2004b), diversas ações de saúde vêm sendo direcionadas a esses segmentos populacionais de forma mais sistemática no SUS, como parte dos esforços intergovernamentais para redução dos indicadores materno e infantil (BRASIL, 2011g). A partir do entendimento de que as morbimortalidades materna e infantil são eventos complexos e, portanto, multifatoriais, essas questões permanecem como um desafio para o país.

Alguns fatores têm dificultado a melhoria desses indicadores, tais como: o financiamento insuficiente; a deficiente regulação do sistema de saúde; a fragmentação das ações e dos serviços de saúde; a organização dos serviços (incluindo a gestão do trabalho das equipes de saúde); e, ainda, a produção do cuidado, que tende a "medicalizar" e "intervir" desnecessariamente nos processos de gestação, parto e nascimento (BRASIL, 2011g).

Considerando o exposto, a saúde materno-infantil vem despontando como uma área governamental com capacidade de mobilização de recursos institucionais. Em 2011, o MS lançou a Rede Cegonha (RC), normatizada pela Portaria no 1.459 , com o objetivo de ampliar o acesso e melhorar a qualidade da atenção pré-natal, a assistência ao parto e ao puerpério e a assistência à criança com até 24 meses de vida (BRASIL, 2011e). No mesmo ano, a Portaria no 1.473 do MS instituiu Comitês Gestores, Grupos Executivos, Grupos Transversais e os Comitês de Mobilização Social e de Especialistas, com atribuições que contribuem na implantação, mobilização de gestores e monitoramento e avaliação da estratégia (BRASIL, 2011f). 
A RC está inserida na discussão de Rede de Atenção em Saúde (RAS). O objetivo da RAS é promover a integração das açōes e serviços de saúde para possibilitar uma atenção eficiente e de qualidade em todos os pontos de atenção, com foco na satisfação dos usuários, e a melhoria dos indicadores de morbimortalidade materno infantil (BRASIL, 2011g).

Para delimitar seu campo de atuação prioritário, a RC parte do diagnóstico de que as mortalidades materna e infantil permanecem elevadas, prevalecendo uma intensa medicalização do nascimento e um uso de tecnologias sem evidências científicas (cesáreas e intervenções desnecessárias no parto) (BRASIL, 2011g). Tesser (2006) define a medicalização como "um processo de expansão progressiva do campo de intervenção da Biomedicina por meio da redefinição de experiências e comportamentos humanos como se fossem problemas médicos".

Esse fenômeno de intensa medicalização do processo do nascimento, somado ao acúmulo de conhecimento e desenvolvimento tecnológico, com persistência de elevadas taxas de morbimortalidade materna, perinatal e infantil, é definido por Diniz (2009) como paradoxo perinatal brasileiro. Isso aponta para uma necessária reorientação do modelo de atenção ao pré-natal, parto, nascimento e puerpério, no qual a incorporação tecnológica seja balizada pelas necessidades das mulheres e crianças, a partir de evidências científicas concretas.

O fato de que o consumo de procedimentos não impacta, necessariamente, nos níveis de saúde é reforçado pela questão de que o acesso ao pré-natal é praticamente universal no país, sem, no entanto, apresentar qualidade satisfatória (BRASIL, 2011g). O modelo de atenção ao pré-natal tem incorporado crescentes custos gerados pela inclusão acrítica de tecnologias (exames, procedimentos, medicamentos); porém, a contrapartida dos resultados não tem sido suficiente (OLIVEIRA NETO, 2009).

Segundo o MS (2010), em 2007, o pré-natal alcançou $89 \%$ de cobertura, e a captação precoce das gestantes foi de $83,6 \%$ (primeira consulta do pré-natal no primeiro trimestre). No entanto, as mortes causadas por síndromes hipertensivas e por causas perinatais e a incidência de sífilis congênita evidenciam a necessidade de qualificar o pré-natal. Essas ocorrências permitem refletir que o pré-natal tem se transformado, em grande medida, num ato burocrático. Outra questão importante é que o Brasil continua com elevado número de partos cirúrgicos 
(cesáreas) em relação aos outros países. Os percentuais revelam que as cesáreas aumentaram de 40\%, em 1996, para 46,5\% em 2007.

O marco conceitual, o discurso técnico-político $e$ as formas de operacionalização da $\mathrm{RC}$ resultam de esforços importantes e de iniciativas de âmbito nacional no campo materno-infantil no SUS - como as diretrizes do Pacto pela Saúde, o Pacto Pela Redução da Mortalidade Materno-Infantil (e o Programa de Qualificação das Maternidades) - e de consistentes experiências municipais e estaduais (BRASIL, 2011g).

A RC prevê ações para a melhoria do acesso e da qualidade da assistência à mulher e à criança, por meio da vinculação da gestante à unidade de referência para o parto e o transporte seguro e da implementação de boas práticas na atenção ao parto e nascimento, incluindo o direito a um acompanhante de livre escolha da mulher no momento parto (BRASIL, 2011g; 2011e). As ações estão inseridas em quatro componentes: (1) Pré-Natal; (2) Parto e Nascimento; (3) Puerpério e Atenção Integral à Saúde da Criança e (4) Sistema Logístico, Transporte Sanitário e Regulação (BRASIL, 2011g; 2011e).

No processo de mobilização para a RC constam tarefas importantes e desafiadoras para os gestores: fomentar a gestão participativa e democrática; promover alinhamento político-institucional e teórico-conceitual das diretrizes para formulação, implementação e monitoramento da RC, culminando com a construção do Modelo Lógico (ML) para avaliação dos resultados alcançados com a implantação da estratégia (BRASIL, 2011f).

O ML é uma ferramenta bastante útil, que permite de maneira visual e sistemática apresentar as relações entre recursos necessários, intervenções (atividades planejadas) e efeitos esperados (produtos, resultados e impactos) que se pretende alcançar com o programa (BEZERRA, CAZARIN e ALVES, 2010).

De acordo com Hartz (1999), o ML busca explicitar a teoria de um programa. Esse instrumento descreve, de forma resumida, o desenho do funcionamento do programa e seus principais componentes, permitindo verificar se este está estruturado para alcançar o desempenho esperado. O ML é uma representação visual e sistemática do programa, que apresenta em forma de figura (tabela, fluxograma) a sequência de passos e relações que conduzem aos efeitos esperados (BEZERRA; CAZARIN; ALVES, 2010; HARTZ; SILVA, 2005; W. K. 
KELLOGG FOUNDATION, 2001). Alguns autores (HARTZ; SILVA, 2005; ROWAN, 2000) apontam a existência de vários formatos de ML, não havendo uma forma certa, errada ou única para sua construção. Segundo Fundação Kellogg (2001), a escolha deve ser realizada de acordo com a forma que melhor se encaixa no programa em razão da informação/mensagem que se precisa passar.

É possível identificar diversas vantagens na utilização do ML: (i) Possibilitar a comunicação do propósito fundamental do programa, evidenciando seus produtos e efeitos esperados (FERREIRA; CASSIOLATO; GONZALEZ, 2007); (ii) Ilustrar a consistência lógica interna do programa, contribuindo para identificar lacunas e efeitos não realísticos (CASSIOLATO; GUERESI, 2010); (iii) Envolver os atores e promover o diálogo sobre o programa entre os envolvidos (financiadores, executores, representantes dos usuários, etc., inclusive avaliadores) (BEZERRA; CAZARIN; ALVES, 2010); (iv) Contribuir para o monitoramento do progresso do programa ao fornecer um plano claro de acompanhamento, de forma que os sucessos possam ser reproduzidos e os problemas evitados; (v) Direcionar as atividades de avaliação do programa ao identificar questōes avaliativas apropriadas e dados relevantes (BEZERRA; CAZARIN; ALVES, 2010; HARTZ, 1999; HARTZ; SILVA, 2005; CASSIOLATO; GUERESI, 2010).

A fim de contribuir com os gestores federais, estaduais e municipais para o cumprimento dessa etapa essencial, este trabalho pretende apresentar uma proposta de ML adaptado às características da RC. Segundo Ferreira, Cassiolato e Gonzalez (2007), o Brasil vem utilizando essa metodologia desde 2007 em diversos órgãos federais, com o objetivo de aperfeiçoar os programas governamentais do PPA 2008-2011.

\section{Metodologia}

Este trabalho utilizou a pesquisa documental para melhor compreender a teoria que embasa a estratégia RC. Foram realizadas consultas a documentos produzidos pelo MS (site oficial do MS, Diário Oficial da União e Comitê Gestor da RC), por outras instituições [Conselho Nacional de Secretários de Saúde (CONASS) e Conselho de Secretários Municipais de Saúde (COSEMS)] e pelos meios de comunicação na internet. 
Utilizou-se o termo "Rede Cegonha" na ferramenta de busca do Google® e no site do MS, no período entre fevereiro e outubro de 2011. Esse período foi selecionado em razão do momento inicial de formulação e lançamento da estratégia.

Foram selecionados para análise seis documentos e uma página do site oficial do MS, conforme descrito a seguir:

- Manual Prático da Rede Cegonha (BRASIL, 2011g);

- Portaria no 1.459, de 21 de junho de 2011 (BRASIL, 2011e);

- Portaria no 650, de 05 de outubro de 2011 (BRASIL, 2011b);

- Nota Técnica: Rede Cegonha (BRASIL, 2011a);

- Página oficial da internet do MS (BRASIL, 2011c);

- Nota Técnica do CONASS No 17/2011: Rede materno-infantil/Rede Cegonha (CONASS, 2011);

- Rede Cegonha COSEMS do Rio de Janeiro (COSEMS, 2011)

Para a construção desta proposta de ML para a RC, foram utilizadas duas referências em modelagem de programas: Bezerra, Cazarin e Alves (2010) e Cassiolato e Gueresi (2010). Após seleção do material de análise, buscaram-se respostas para o conjunto de perguntas-chave definidas por Bezerra, Cazarin e Alves (2010) como norteadoras para a construção de programas e políticas de saúde. Segundo as autoras, "para começar a delinear um programa é importante responder alguns questionamentos que explicitam seus aspectos essenciais". A utilização das perguntas-chave como guia permitiu entrar em contato com questôes fundamentais relativas ao passo a passo da estratégia.

As 12 perguntas-chave utilizadas neste estudo são: (1) Qual o problema visado pelo programa, pelos seus formuladores federais e executores ou agentes locais? (2) Qual o programa de saúde criado para resolver o problema? (3) Qual o objetivo geral do programa? (4) Quais são seus objetivos específicos? (5) Quais as metas que o programa pretende alcançar? (6) Qual é sua população-alvo? (7) Quais são os componentes do programa? (8) Que atividades são realizadas? (9) Quais as estruturas de que o programa precisa para funcionar? (10) Quais os produtos que se espera obter com a realização das atividades? (11) Quais os resultados que 
o programa pretende alcançar? (12) Quais os fatores que podem influenciar no alcance desses resultados, que não apenas os relacionados ao programa?

Em seguida, utilizou-se a proposta de Cassiolato e Gueresi (2010), que define três componentes para o ML: (1) Explicação do problema e referências básica; (2) definição dos fatores de contexto; e (3) estruturação do programa para alcance de resultados. Neste estudo, apenas o primeiro componente foi utilizado, o que resultou na construção da "Árvore de Problemas" (Figura 1), em razão desse recurso permitir mostrar de forma clara os problemas. Por fim, foi realizada a diagramação de um ML para a estratégia RC.

Será utilizado neste trabalho o termo "elementos" como sinônimo de "componentes" do ML, visando evitar confusão com os componentes da estratégia RC definidos pelo MS. Dessa forma, o ML proposto terá como elementos: objetivos gerais, componentes da estratégia $\mathrm{RC}$, ações e indicadores.

A etapa de validação desse Modelo Lógico para a estratégia não foi realizada, tendo em vista ser esta uma proposta que deverá ser adaptada à realidade de cada estado/município e posteriormente validada.

\section{Resultados}

A elaboração do ML da estratégia RC seguiu os seguintes passos:

- Resposta às 12 perguntas-chave propostas por Bezerra, Cazarin e Alves (2010), que resultaram no quadro 1 ;

- Explicação do problema e referências básicas a partir da adaptação da figura desenvolvida pelo MS, derivando na figura 1;

- Construção do produto final: a proposta de ML da RC.

No quadro 1, observa-se que os documentos selecionados responderam a oito das 12 perguntas-chave propostas por Bezerra, Carazin e Alves (2010): 1) referente ao problema; 2) relacionada aos programas existentes para solucionar o problema; 3) relativa ao objetivo geral; 5) referente às metas; 6) sobre a população-alvo; 7) relacionada aos componentes do programa; 8 ) relativa às atividades do programa; e 9) referente às estruturas para funcionamento do programa. Nos documentos selecionados não constam, claramente, as respostas para as perguntas-chave: 4) relativa aos objetivos específicos; 10) relacionada aos produtos esperados; 11) 
referente aos resultados pretendidos; e 12) sobre os fatores que podem influenciar no resultado da estratégia RC.

As perguntas-chave criam uma sequência capaz de trazer à tona as interrelações existentes na teoria do programa, facilitando a construção do desenho do ML. No caso da RC, elas revelam questôes essenciais: o problema que a estratégia pretende enfrentar, as metas, que são os objetivos específicos quantificados e remetem a um determinado tempo, os objetivos específicos que se relacionam às atividades previstas no programa, metas, população-alvo, componentes, estrutura para funcionamento da estratégia e os resultados a serem alcançados.

Dessa forma, as perguntas respondidas possibilitam traçar as relações existentes nos elementos da RC. Quando a resposta para a pergunta-chave 1 é obtida, o problema existente vem à tona. Em seguida, a reflexão que se tem ao responder a pergunta-chave 3 (objetivo geral) refere-se à mudança que se quer para o problema. As ações (pergunta-chave 8) que integram a estratégia e pretendem resolver o problema fazem conexão com os recursos (pergunta-chave 9) necessários para desenvolvê-las, e buscam alcançar os resultados (perguntachave 11). Finalmente, as metas definidas (pergunta-chave 5) devem estar compatíveis com os recursos disponíveis.

Segundo Cassiolato e Gueresi (2010), quando o problema a ser enfrentado é definido, torna-se mais fácil identificar o objetivo geral, que aponta para a mudança da situação do problema. Como consequência, a população-alvo fica evidente e ficam mais claras as ações que irão integrar o programa. Essas ações geram produtos a serem ofertados aos beneficiários do programa. Por fim, os resultados intermediários destacam as mudanças nas causas do problema levando ao resultado esperado (diretamente relacionado ao objetivo do programa), refletindo a mudança no problema.

Os objetivos específicos não foram respondidos (pergunta-chave 4); porém, devem servir como diretrizes que norteiam o conjunto das ações (CASSIOLATO; GUERESI, 2010). É fundamental destacar que o resultado alcançado (perguntachave 11) para cada objetivo específico contribui para o alcance do objetivo geral (pergunta-chave 3). Para os produtos esperados, não se obteve resposta. Os produtos são diretamente gerados pelas ações e devem ser claramente definidos, assim como os resultados intermediários advindos deles (CASSIOLATO; GUERESI, 2010). 
Os resultados pretendidos com a estratégia, que evidenciam as mudanças nas causas dos problemas, também não foram identificados. Os resultados intermediários congregam os resultados alcançados com produtos do programa que conduzem ao resultado final, o alcance do objetivo do programa (CASSIOLATO; GUERESI, 2010).

Pode-se afirmar que a pergunta-chave 12 não foi respondida por meio dos documentos, principalmente no período analisado, no qual a $\mathrm{RC}$ ainda estava em fase inicial de implantação. Entretanto, foi possível observar que os documentos evidenciam uma aposta na gestão tripartite da RC (representantes do Ministério da Saúde, da Secretaria Estadual da Saúde e do COSEMS), e, ainda, a inclusão de outros atores (profissionais da saúde, movimento de mulheres e sociedade civil) nesse processo (BRASIL, 2011a).

É central ressaltar a importância dos executores locais da RC buscarem conhecer e se apropriar dos fatores de contexto em cada estado ou município, por serem informaçóes peculiares desses locais que apresentam conjuntura bastante diversificada no país, visto que podem influenciar positivamente ou negativamente na implantação da estratégia. Entre esses fatores, estão: contexto político, situação epidemiológica, profissionais de saúde capacitados, movimentos sociais implicados no processo e outros.

Sobre os fatores de contexto, Cassiolato e Gueresi (2010) afirmam que "esse é um dado importante da realidade do programa, o qual irá permitir conhecer a sustentabilidade das hipóteses assumidas na sua estruturação lógica para o alcance de resultados". A falta desse elemento tão valioso dificulta a transmissão do que é e do que se pretende com a RC, podendo originar várias dúvidas, falta de consenso entre os envolvidos e, com isso, gerar entraves na sua implantação nas localidades. 
Quadro 1. Apresenta as perguntas-chave propostas por Bezerra, Cazarin e Alves

(2010) para a construção do Modelo Lógico e as respostas encontradas nos documentos selecionados neste estudo sobre a Rede Cegonha

\begin{tabular}{|c|c|c|c|c|c|c|c|}
\hline \multirow[b]{2}{*}{ PERGUNTAS CHAVES } & \multicolumn{7}{|c|}{ DOCUMENTOS PRODUZIDOS PELA REDE CEGONHA } \\
\hline & 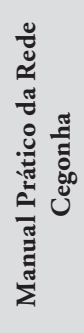 & 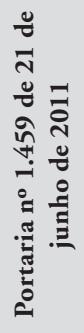 & 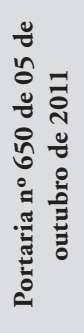 & 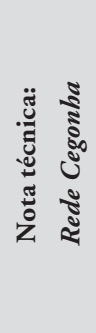 & 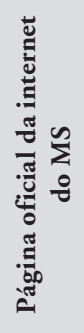 & 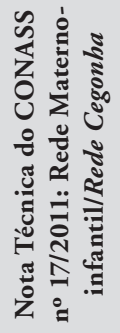 & 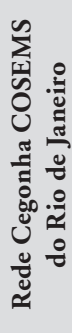 \\
\hline $\begin{array}{l}\text { 1. Qual o problema visado pelo } \\
\text { programa, pelos seus formuladores } \\
\text { federais e executores ou agentes } \\
\text { locais? }\end{array}$ & $\mathrm{X}$ & $\mathrm{X}$ & - & $\mathrm{X}$ & - & - & - \\
\hline $\begin{array}{l}\text { 2. Qual o programa de saúde criado } \\
\text { para resolver o problema? }\end{array}$ & $\mathrm{X}$ & $\mathrm{X}$ & - & $\mathrm{X}$ & $\mathrm{X}$ & - & $\mathrm{X}$ \\
\hline $\begin{array}{l}\text { 3. Qual o objetivo geral do } \\
\text { programa? }\end{array}$ & $\mathrm{X}$ & $\mathrm{X}$ & - & $\mathrm{X}$ & $\mathrm{X}$ & $\mathrm{X}$ & $\mathrm{X}$ \\
\hline $\begin{array}{l}\text { 4. Quais são seus objetivos } \\
\text { específicos? }\end{array}$ & - & - & - & - & - & - & - \\
\hline $\begin{array}{l}\text { 5. Quais as metas que o programa } \\
\text { pretende alcançar? }\end{array}$ & $\mathrm{X}$ & - & $\mathrm{X}$ & - & - & - & - \\
\hline 6. Qual é sua população alvo? & $\mathrm{X}$ & $\mathrm{X}$ & $\mathrm{X}$ & $\mathrm{X}$ & $\mathrm{X}$ & $\mathrm{X}$ & $\mathrm{X}$ \\
\hline $\begin{array}{l}\text { 7. Quais são os componentes do } \\
\text { programa? }\end{array}$ & $\mathrm{X}$ & $\mathrm{X}$ & $\mathrm{X}$ & $\mathrm{X}$ & $\mathrm{X}$ & $\mathrm{X}$ & $\mathrm{X}$ \\
\hline $\begin{array}{l}\text { 8. Que atividades são realizadas no } \\
\text { programa? }\end{array}$ & $\mathrm{X}$ & $\mathrm{X}$ & $\mathrm{X}$ & $\mathrm{X}$ & - & - & - \\
\hline $\begin{array}{l}\text { 9. Quais as estruturas de que o } \\
\text { programa precisa para funcionar? }\end{array}$ & $\mathrm{X}$ & $\mathrm{X}$ & $\mathrm{X}$ & $\mathrm{X}$ & $\mathrm{X}$ & - & $\mathrm{X}$ \\
\hline $\begin{array}{l}\text { 10. Quais os produtos que se } \\
\text { espera obter com a realização das } \\
\text { atividades? }\end{array}$ & - & - & - & - & - & - & - \\
\hline $\begin{array}{l}\text { 11. Quais os resultados que o } \\
\text { programa pretende alcançar? }\end{array}$ & - & - & - & - & - & - & - \\
\hline $\begin{array}{l}\text { 12. Quais os fatores que podem } \\
\text { influenciar no alcance destes } \\
\text { resultados, que não apenas os } \\
\text { relacionados ao programa? }\end{array}$ & - & - & - & - & - & - & - \\
\hline
\end{tabular}

Fontes: Adaptado pela autora com base em Guerrero (2010). 
A figura na qual o problema é exposto foi adaptada de outra produzida pelo MS (BRASIL, 2011c). Optou-se por utilizar um documento do MS porque mostra o problema no entendimento do grupo formulador da estratégia. Na adaptação realizada neste artigo, foram inseridas informaçôes adicionais sobre a Rede Cegonha, seus objetivos, sua população-alvo e seus beneficiários. Na figura 1, estão explicitados os problemas, suas causas e consequências, e a estratégia formulada pelo MS (Rede Cegonha) para enfrentar esses problemas de forma estruturada.

Para Cassiolato e Gueresi (2010), a construção da "Árvore de problemas" é um dos passos de pré-montagem do Modelo Lógico. Esse método tem sido muito utilizado pelo Governo Federal para elaborar programas do Plano Plurianual.

Por fim, na figura 2, tem-se a proposta de um ML para a RC que fornece uma representação visual dessa estratégia, explicitando seus principais "elementos": objetivos gerais, componentes, ações e indicadores. Demonstra-se, a partir dessa iniciativa, que a RC é um recorte materno-infantil da política de saúde da mulher e da criança, não se caracterizando como um programa; por isso o termo Estratégia $R C$ foi adotado neste estudo.

A estratégia diz respeito a um conjunto de ações prioritárias do Governo Federal e, consequentemente, dos executores locais, que visam à melhoria da atenção dirigida às mulheres e às crianças.

Observa-se no ML que o Planejamento Familiar (PF) não foi elencado como um componente da estratégia $\mathrm{RC}$. O PF, no contexto dos direitos reprodutivos, tem o objetivo de garantir às mulheres e aos homens o direito de ter ou não filhos. Faz parte das ações da Política de Assistência Integral à Saúde da Mulher preconizada pelo Ministério da Saúde. Portanto, os serviços devem garantir acesso aos meios para evitar ou propiciar a gravidez, acompanhamento clínico e açôes educativas, visando escolhas conscientes (BRASIL, 2002).

A gravidez indesejada é apontada pelo MS na "árvore de problemas" (quadro 1) como uma questão que provoca mortes de mulheres e crianças. Todavia, as ações que buscam enfrentar esse problema estão inseridas nos componentes do pré-natal (programas educativos relacionados à saúde sexual e reprodutiva) e do puerpério (orientação e oferta de métodos contraceptivos). Na RC, as ações de educativas e os métodos contraceptivos serão apenas para as mulheres que já estão grávidas ou no puerpério, não estando direcionadas a outros grupos de mulheres que podem ser alvo das ações do PF. 


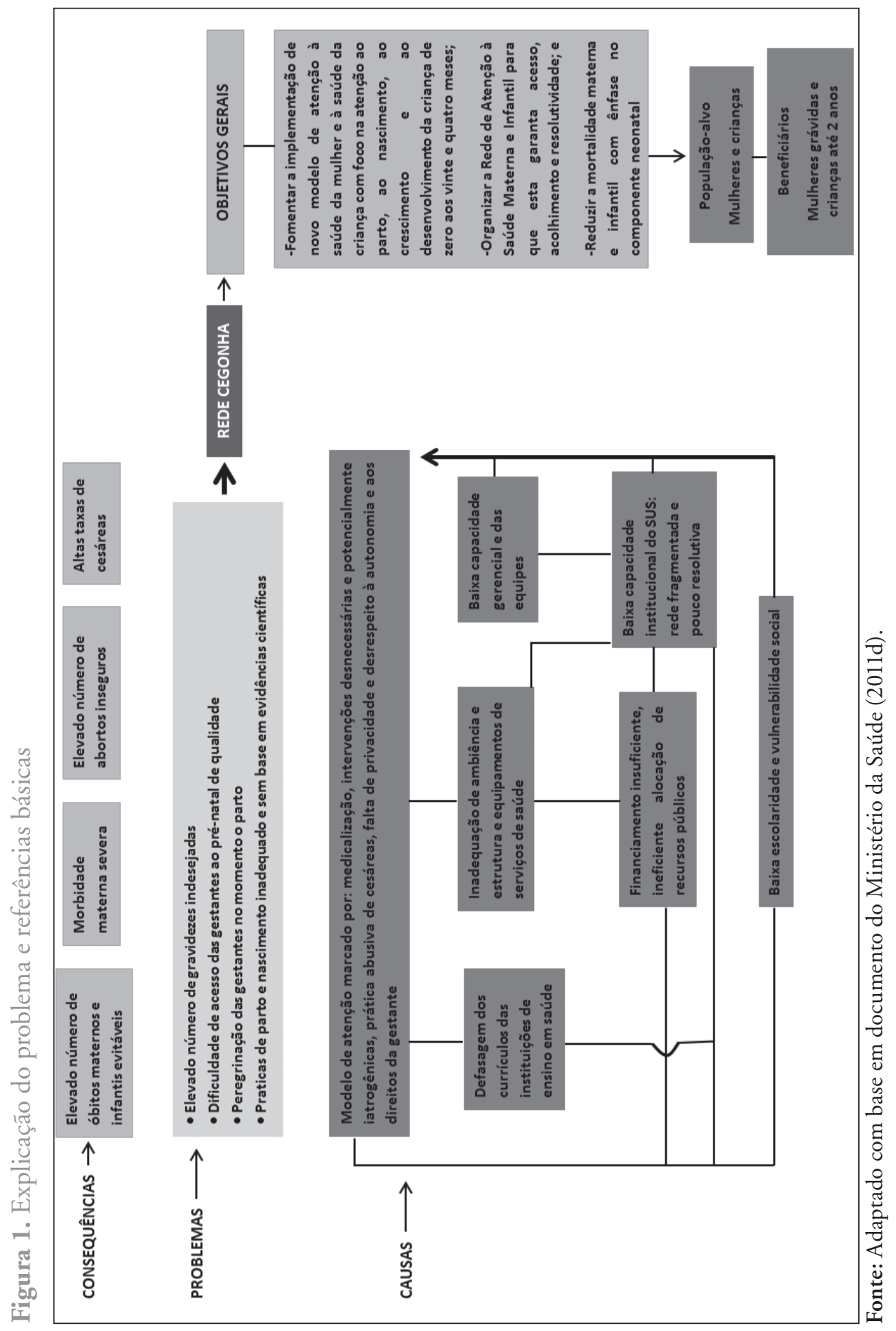




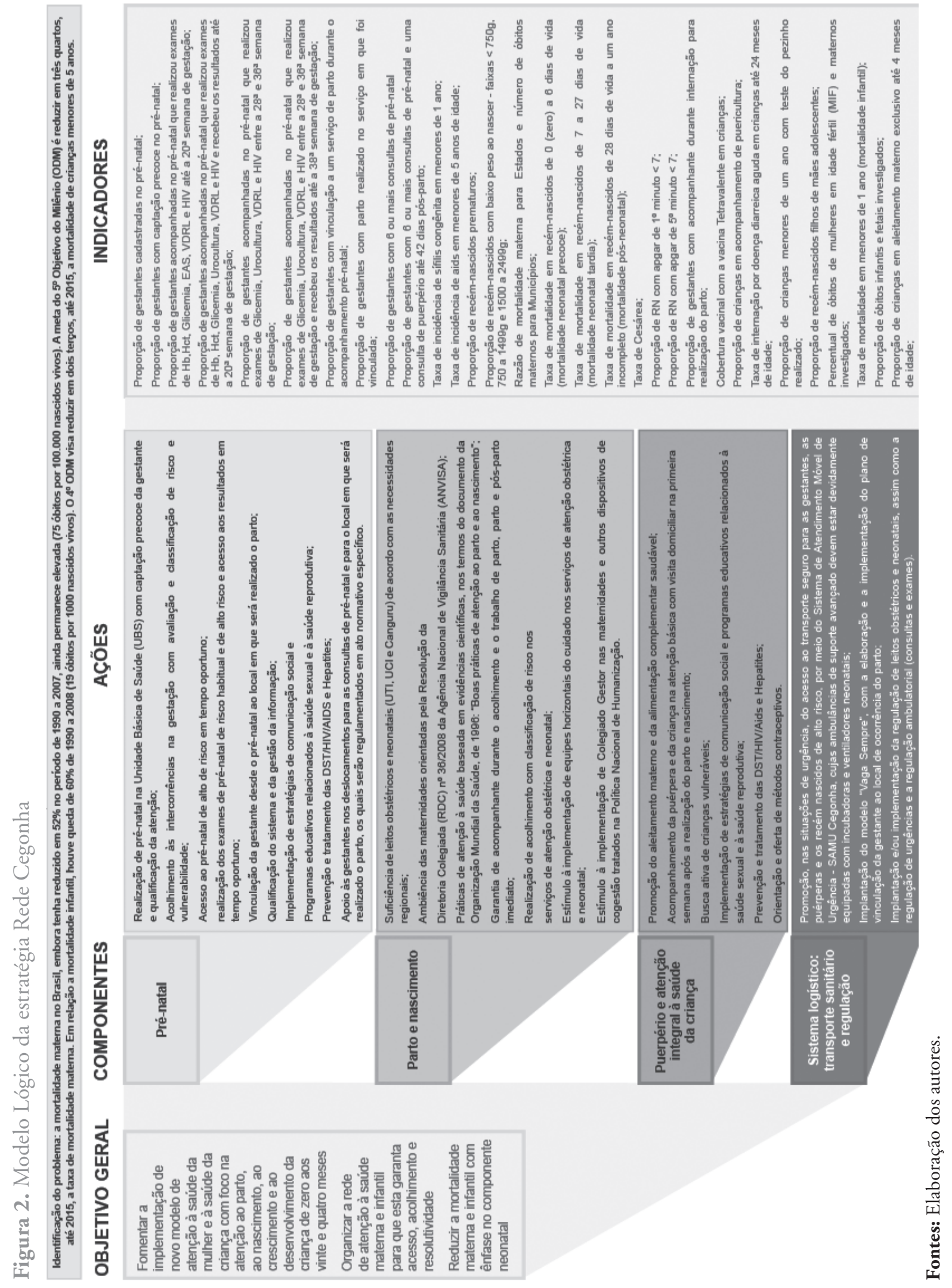


Como sugestão para a RC no que diz respeito ao $\mathrm{PF}$, é fundamental serem realizadas novas discussões, trazendo uma reformulação/ajuste na teoria dessa estratégia e aumentando suas chances de sucesso. Isso porque se trata de um tema que aborda ações que promovem a maternidade/paternidade responsáveis, com participação de mulheres e homens nas ações de pré-natal e do parto/nascimento, de forma natural, como corresponsáveis.

Dentre os indicadores que o MS elencou para monitoramento da estratégia, chamam a atenção o "percentual de óbitos de mulheres em idade fértil e maternos investigados", e a "proporção de óbitos infantis e fetais investigados". Isso porque não foram previstas ações voltadas para a vigilância de óbitos maternos e infantis, principalmente no que diz respeito ao fortalecimento dessas açóes no âmbito das equipes de atenção básica. Ainda, nos documentos analisados, não ficou evidente a importância da articulação das ações da $R C$ com os Comitês de Mortalidade Materna, nem com os Comitês de Prevenção do Óbito Infantil. Trazer essa discussão à tona é essencial, pois as ações que objetivam reduzir esses óbitos não devem estar isoladas, possibilitando maior capacidade de reduzir as taxas de mortalidade.

\section{Considerações finais}

A RC, proposta pelo Governo Federal, é uma estratégia que visa enfrentar o problema do elevado número de óbitos de mulheres e de crianças por meio de iniciativas que mudem esse cenário existente no Brasil. As açôes dessa estratégia vão além do repasse de recursos atrelado ao aumento de leitos ou da oferta de procedimentos: elas buscam a melhoria da atenção destinada a sua população-alvo.

Essa iniciativa convoca os gestores, os trabalhadores e a sociedade a refletir e transformar o modelo de atenção ao parto e ao nascimento praticado no país, o qual não coloca a gestante/parturiente no centro da "cena", como protagonista do processo de gestação e parto, medicalizando e intervindo muitas vezes desnecessariamente. Nos documentos analisados, fica evidente que há o entendimento por parte dos formuladores da RC de que a mulher deve estar no centro no processo, implicada e vivendo a experiência da gravidez, do parto e da maternidade com segurança.

Apesar disso, a RC não definiu ações de planejamento familiar considerando as mulheres e seus parceiros/parceiras que não desejam ter filhos; porém, os direitos reprodutivos contemplam os de querer ou não ter filhos. As mulheres 
1312 deixam de ser sujeitos principais no evento reprodutivo, de estar no centro do processo. Só depois de engravidar isso deverá ser considerado pela RC.

Outra questão fundamental para debate acerca da rede é a perspectiva de que os "pontos de atenção" necessários ao cuidado materno-infantil devem estar conectados e funcionar em rede, minimizando, assim, a fragmentação, o desperdício de recurso e a ineficiência, ao mesmo tempo em que busca garantir a integralidade da assistência.

O presente ML mostra-se útil como uma ferramenta de gestão, com papel de explicitar a estratégia de forma prática e clara, e, também, auxiliar o processo de comunicação e divulgação da estratégia em oficinas da RC com os gestores dos estados ou municípios, trabalhadores de saúde e junto à população. Ao explicitar a teoria do programa, o modelo pode ser utilizado na organização dos trabalhos de avaliação.

A construção deste ML da RC permitiu identificar os atores-chave e seus papéis, os objetivos, as açôes, as metas, os recursos e os indicadores da RC, que é uma das prioridades do Governo Federal.

Um ML com o desenho da RC é importante para que os atores envolvidos (gestores, profissionais de saúde, população, mídia) possam compreender e debater suas questões com base em uma figura que sintetiza e explicita objetivamente pontos importantes da estratégia, principalmente no momento de sua implantação, no qual existem muitas dúvidas, incertezas e diversos documentos (oficiais e não oficiais) que não abordam o tema de forma clara e detalhada.

A RC é uma oferta do MS para os estados e municípios, mas são os gestores municipais que aderem à estratégia, ou seja, que decidem se no município as ações previstas na RC serão desenvolvidas em nível local.

É oportuno lembrar que, por ter sido formulada em nível federal, nos documentos selecionados não há informações acerca dos fatores de contexto. Tendo em vista que a RC será estruturada em regiōes com características distintas, é fundamental que o Comitê Gestor consiga descrever esses fatores conforme prevê a Portaria no 1.473 , de 24 de junho de 2011. Vale destacar a importância de conhecer os fatores de contexto (questões políticas, situação epidemiológicas, recursos financeiros, materiais e humanos, profissionais capacitados), que podem influenciar no alcance dos resultados esperados, pois podem contribuir ou comprometer a implantação do programa. 
Outro aspecto importante é a existência de lacunas na lógica da estratégia - falta de descrição de seus elementos e não previsão de ações de planejamento familiar e de vigilância dos óbitos de mulheres e crianças. Portanto, é necessário rever a teoria da RC a fim de detalhar e ampliar seu foco e suas ações, o que pode aumentar suas chances de sucesso.

Com base no exposto, acredita-se que esta proposta de ML para a estratégia RC cumpre sua missão de ferramenta útil que pode auxiliar o planejamento, a implantação e a avaliação do cumprimento das ações previstas nos estados e municípios brasileiros. ${ }^{1}$

\section{Referências}

BEZERRA, L. C. de A.; CAZARIN, G.; ALVES C. K. de A. Modelagem de programas: da teoria à operacionalização. In: SAMICO, I. et al. (Org.). Avaliação em saúde: bases conceituais e operacionais. Rio de Janeiro: MedBook, 2010. p. 65-78.

BRASIL.Ministério da Saúde. Secretaria de Políticas de Saúde. Área Técnica de Saúde da Mulher. Assistência em Planejamento Familiar: manual técnico. 4. ed. Brasília, 2002.

. Ministério da Saúde. Nota téenica: Rede Cegonha. Brasília: 2011a.

. Ministério da Saúde. Parto, Aborto e Puerpério: assistência humanizada a mulher. Brasília: MS, 2001.

. Ministério da Saúde. Portaria no 569, de 1 de junho de 2000. Institui o Programa de Humanização no Pré-natal e Nascimento, no âmbito do Sistema Único de Saúde - SUS. Diário Oficial da União, Brasília, 2000. Seção 1, p. 4.

. Ministério da Saúde. Portaria no 650, de 5 de outubro de 2011. Dispõe sobre o os Planos de Ação regional e municipal da Rede Cegonha. Diário Oficial da União, Brasília, 2011b. Seção 1.

. Ministério da Saúde. Rede Cegonha. 2011c. Disponível em: http://portal.saude. gov.br/PORTAL/SAUDE/GESTOR/AREA.CFM?ID_AREA=1816. Acesso em: ago 2011.

. Ministério da Saúde. Rede Cegonha: contribuir para a Atenção Integral da Saúde da Mulher e Criança. Brasília: 2011d. 85 slides: coloridos.

Ministério da Saúde. Secretaria de Atenção à Saúde. Departamento de Ações Programáticas Estratégicas. Portaria no 1.459, 24 de junho de 2011. Institui, no âmbito do Sistema Único de Saúde, a Rede Cegonha. Diário Oficial da Uniāo, Brasília, 2011e. Seção 1. . Ministério da Saúde. Secretaria de Atenção à Saúde. Departamento de Ações Programáticas Estratégicas. Portaria no 1.473, 24 de junho de 2011. Instituiu os Comitês Gestores, Grupos Executivos, Grupos Transversais e os Comitês de Mobilização Social e 
de Especialistas dos compromissos prioritários de governo organizados por meio de Redes Temáticas de Atenção à Saúde. Diário Oficial da União, Brasília, $2011 \mathrm{f}$.

- Ministério da Saúde. Secretaria de Atenção à Saúde. Departamento de Ações Programáticas Estratégicas. Políticas atenção integral à saúde da mulher: princípios e diretrizes. Brasília, 2004a.

- Ministério da Saúde. Secretaria de Atenção à Saúde. Manual prático para implementação da Rede Cegonha. Brasília: 2011g.

- Ministério da Saúde. Secretaria de Atenção à Saúde. Pacto pela redução da mortalidade materna e neonatal. Brasília, 2004b. Mimeo.

Ministério da Saúde. Secretaria de Vigilância em Saúde. Departamento de Análise de Situação de Saúde. Saúde Brasil 2009: uma análise da situação de saúde e da agenda nacional e internacional de prioridades em saúde. Brasília: 2010. Disponível em: <http://portal.saude. gov.br/portal/arquivos/pdf/29_11_10_saude_brasil_web.pdf>. Acesso em: jun 2011.

- Ministério da Saúde. Secretaria de Vigilância em Saúde. Departamento de Análise de Situação de Saúde. Saúde Brasil 2008: 20 anos de Sistema único de Saúde (SUS) no Brasil. Brasília: MS, 2009.

CASSIOLATO, M.; GUERESI, S. Como elaborar modelo lógico: roteiro para formular programas e organizar avaliação. Brasília: 2010.

CONSELHO NACIONAL DOS SECRETÁRIOS DE SAÚDE. Rede Cegonha. Brasília: CONASS, 2011.

COSEMS. Conselho de Secretarias Municipais de Saúde. Rede Cegonha. Apresentação realizada pelo Conselho de Secretarias Municipais de Saúde do Rio de Janeiro. Rio de Janeiro: 2011. Disponível em: <http://cosemsrj.org.br/images/rede_cegonha_jul11.pdf>. Acesso: set 2011.

DINIZ, C.S.G. Humanização da assistência ao parto no Brasil: os muitos sentidos de um movimento. Ciência e Saúde Coletiva. Rio de Janeiro, v. 10, p. 627-37, 2005.

Gênero, saúde materna e o paradoxo perinatal. Revista Brasileira Crescimento

Desenvolvimento Humano [online], v. 19, n. 2, p. 313-326. São Paulo, 2009. Disponível em: <http://www.revistasusp.sibi.usp.br/pdf/rbcdh/v19n2/12.pdf>. Acesso: jul 2011.

FERREIRA, H.; CASSIOLATO, M.; GONZALEZ, R. Como elaborar Modelo Lógico de programa: um roteiro básico. Brasília: 2007. Disponível em: <http://agencia.ipea.gov.br/ images/stories/PDFs/TDs/td_1369.pdf>. Acesso em: set 2011.

GUERRERO, A. V. P. Avaliabilidade do pacto pela redução da mortalidade infantil nas regiōes Amazônia legal e nordeste do Brasil: descrição do programa e construção do modelo lógico. 2010. 75p. Dissertação (Mestrado em Saúde Coletiva) - Centro de Pesquisas Aggeu Magalhães, Fundação Oswaldo Cruz, Recife, 2010. 
HARTZ, Z. M.A., SILVA, L.M.V. da. Avaliação em saúde: dos modelos teóricos à prática na avaliação de programas e sistemas de saúde. Salvador: EDUFBA, 2005. 275 p.

HARTZ, Z. M. de A. Avaliação dos programas de saúde: perspectivas teórico-metodológicas e políticas institucionais. Ciência e Saúde Coletiva. Rio de Janeiro, v. 4, n. 2, p. 341-353, 1999. OLIVEIRA NETO, A. V. O Modelo Tecnoassistencial em Saúde na Cidade do Recife no período de 2001-2008: em busca de seus marcos teóricos. 2009. 61p. Monografia (Residência em Medicina de Família e Comunidade) - Faculdade de Ciências Médicas da Universidade de Pernambuco. Recife, 2009.

RATTNER, D. Humanização na atenção a nascimentos e partos: ponderações sobre políticas públicas. Interface (Botucatu) online, v.13, p. 759-768. Botucatu, 2009. Disponível em: <http://dx.doi.org/10.1590/S1414-32832009000500027>. Acesso em: jul 2011.

ROWAN, M. Logic Models in Primary Care Reform: Navigating the Evaluation. The Canadian Journal of Program Evaluation, v. 15, n. 2, p. 81-92. 2000.

TESSER, C. D. Social medicalization (II): biomedical limits and proposals for primary care clinic. Interface (Botucatu) online, v. 10, n. 20, p.1-19. Botucatu, 2006. Disponível em: <http://socialsciences.scielo.org/scielo.php?pid=S1414-32832006000200004\&script=sci_ abstract>. Acesso em: set 2011.

W. K. KELLOGG FOUNDATION. Using logic models to bring together planning, evaluation, \& action: Logic model development guide. Battle Creek (MI): 2001.

\section{Nota}

${ }^{1}$ P.C.S. Cavalcanti participou da concepção do trabalho, planejamento, análise e interpretação dos dados, redação e revisão final do artigo. G.D. Gurgel Junior participou da redação e revisão final do artigo. A.L.R. Vanconcelos e A.V.P. Guerrero participaram da concepção do trabalho, planejamento, análise e interpretação dos dados, redação e revisão final do artigo. 
A logical model of the Rede Cegonha network This paper aimed to build a Logic Model (LM) of the "Rede Cegonha Network", which was launched by the Health Ministry in 2011, to ensure qualified care for pregnant women and children up to two years old. The network points out that both maternal and child morbidity and mortality remains high, with prevalence of the medicalization of birth and use of technologies without scientific evidence and does not consider pregnant women as protagonists of their pregnancy and birthing process. A documentary research was carried out on official documents and other sources within the Health Ministry, between February and October 2011. The preparation of the LM was divided in three stages: answer twelve "key questions" for modeling programs; explanation of the problem and basic references, and construction of the LM of the network based on Bezerra, Cazarin and Alves (2010) and Cassiolato and Gueresi (2010) modeling programs. "Gaps" were observed in the network's logic, pointing to the need to revise the theory of this strategy in order to increase the chances of success. The LM has proven useful as a management tool, to clarify the role of the network practice and to assist both the dissemination and the communication process, and can also be used to organize assessment studies.

> Key words: maternal mortality; infant mortality; Logical Model; Rede Cegonha network. 